\title{
Forward backward multiplicity correlations: a tool to study pro- perties of the proton-proton collisions
}

\author{
Edgar Dominguez-Rosas ${ }^{1, \star}$ and Eleazar Cuautle Flores ${ }^{1, \star \star}$ \\ ${ }^{1}$ Instituto de Ciencias Nucleares, Universidad Nacional Autónoma de México. Apartado Postal 70-543, \\ Ciudad de México 04510, México
}

\begin{abstract}
Forward-backward multiplicity correlations have been used to study hadron production mechanisms in electron-positron, proton-proton and more recently in leadlead collisions. The experimental results on this correlations and its comparison to different models reveals an incomplete agreement. In this work, we present an study of forward backward multiplicity correlations in proton-proton collisions using PYTHIA event generator, at LHC energies. Detailed analysis is presented in the case of soft and hard QCD processes, incorporating color reconnection model as part of hadronization mechanism and multiple parton interactions effects in the correlations. Our results and its comparison to available experimental data suggest that this kind of correlations are great tools to characterize the events and gives the possibility to disentangle phenomena in hard and soft QCD processes.
\end{abstract}

\section{Introduction}

The Forward Backward (F - B) multiplicity correlations had been studied long time ago, on different colliding system. One of the first results in $\mathrm{p}+\mathrm{p}$ collisions in ISR at $\sqrt{s}=52.6 \mathrm{MeV} / c$ [1] where was found positive values for the correlations, five years later there were results of $\mathrm{p}+\overline{\mathrm{p}}$ collisions at $\sqrt{s}=570 \mathrm{MeV} / c$ [2] in $S P S$, again positive values of the correlation were found, and a possible dependence on the energy was reported. Following this kind of correlations, results on $e^{+} e^{-}$collisions at $\sqrt{s}=29 \mathrm{GeV}$ [3] were published, the reported results shows no $\mathrm{F}-\mathrm{B}$ correlations, this result was interpreted as a consequence of the independent fragmentation of the jets produced in the collision. Few years later, results of the E735 collaboration at Tevatron [4] in $\mathrm{p}+\mathrm{p}$ collision at $\sqrt{s} \approx 1 \mathrm{TeV}$ confirmed the dependence of the correlation with the energy.

Results on heavy ions at $\sqrt{s_{N N}}=200 \mathrm{GeV}$, published by the STAR Collaboration at RHIC [5], found strong correlations in case of $\mathrm{Au}+\mathrm{Au}$ collisions for centrality $0-10 \%$, meanwhile for $\mathrm{p}+\mathrm{p}$ collisions was found very low correlation. Recently at the LHC were published similar results in $\mathrm{p}+\mathrm{p}$ collision by ATLAS [6] and ALICE [7] collaboration at $\sqrt{s}=0.9,2.7,9 \mathrm{TeV}$, they observed strong correlations. Their analysis was more detailed investigating the $p_{\mathrm{T}}$ dependence of azimuthal and pseudorapidity distributions of $\mathrm{F}-\mathrm{B}$ multiplicity correlation.

\footnotetext{
$\star$ e-mail: edgar.dominguez.rosas@ cern.ch

$\star \star$ e-mail: ecuautle@nucleares.unam.mx
} 
In the present work we analyze $\mathrm{F}-\mathrm{B}$ multiplicity correlations and possible effects on it due to final state interactions in the hadronization mechanism, those like color reconnection (CR) as well as from the number of multiple parton interactions (nMPI), these phenomenological models are successfully used to describe kinematic variables like average transverse momentum of charged hadrons and multiplicity distributions, hence its importance to investigate the effects on $\mathrm{F}-\mathrm{B}$ multiplicity correlations.

The F-B multiplicity correlation strength is defined as correlation coefficient of forward and backward multiplicity, where $n_{F}$ and $n_{B}$ are the numbers of charged particles falling into the forward and backward pseudorapidity intervals of $\delta \eta$, respectively:

$$
b_{\text {Corr }}=\frac{\left\langle n_{F} n_{B}\right\rangle-\left\langle n_{F}\right\rangle\left\langle n_{B}\right\rangle}{\left\langle n_{F}^{2}\right\rangle-\left\langle n_{F}\right\rangle^{2}},
$$

this strength of the correlation factor, $b_{\text {corr }}$, is really sensitive to the changes of multiplicity, for instance, for ranges of multiplicity is possible to get negative values of $b_{\text {corr }}$, this results are consequences of the definition, but those results have no physical meaning.

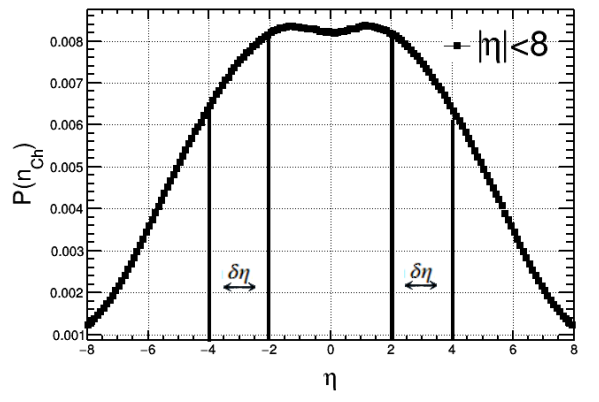

Figure 1: Pseudorapidity distribution illustrating $\delta \eta$ in forward-backward region
In the Fig. 1 shows the pseudorapidity distribution, where is illustrated the $\delta \eta$ variable which is the interval of the multiplicity to measure the correlation. This variable allow to study the out coming hadrons of the collisions, those are expected to come from the collisions if we analyze central pseudorapidity regions, while for larger values of $\eta$ we expect to see hadrons coming from the fragmentation region, and also from colliding beam.

\section{Forward-Backward multiplicity correlation with PYTHIA}

The analysis of the correlation is realized with Monte Carlo event generator, PYTHIA 8.2 [8], taking into account soft and hard QCD process, with a sample of 25 billions of events for each set of studies, selecting charged primary hadrons with transverse momentum in the range $0.3<p_{\mathrm{T}}<1.5$ $\mathrm{GeV} / c$ and pseudorapidity range $|\eta|<1$. These values are taken to be able to compare our results with those presented by the ALICE experiment [7].

Pseudorapidity distribution is the first variable to be understood since any change on it, will bring consequences on the correlations. It is important to quantify the effects of soft and hard QCD processes in an independent way. The left panel of the Fig. 2 shows these two distributions generated in $\mathrm{p}+\mathrm{p}$ collisions in a width $\eta$ range showing a higher number of charged hadrons produced in the central region for hard processes while the opposite is observed for large $\eta$ values (fragmentation region). The differences could be understood in terms of the kind of processes occurred in the collisions, it means, hard processes mean harder collision between interaction partons, producing jets in transverse direction respect to that of the beam. Right panel of the figure shows the transverse momentum distribution for soft and hard QCD processes, from which one sees a spectrum hardest for hard QCD processes, also it shows a spectrum for central rapidity compared to fragmentation 

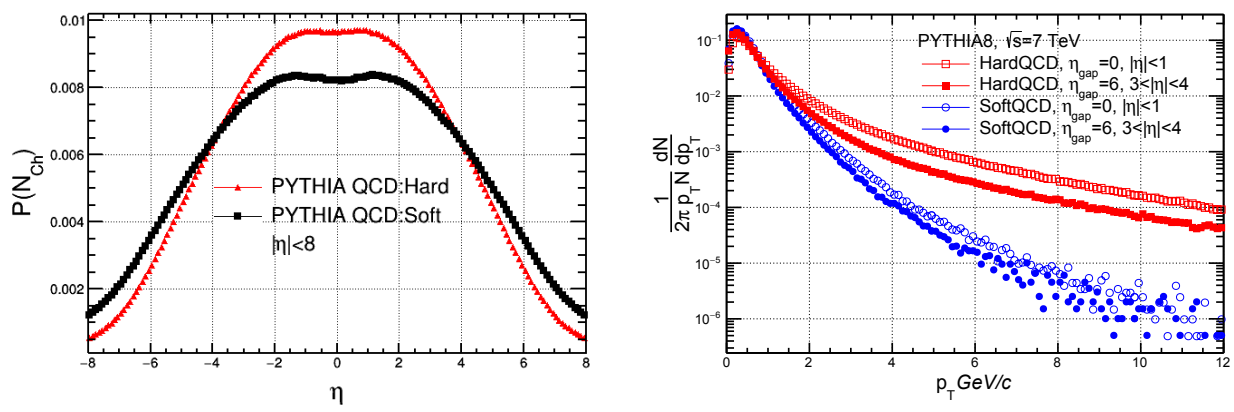

Figure 2: Pseudorapidity distributions (left) for soft and hard QCD processes and transverse momentum distributions (right) at $\sqrt{s}=7 \mathrm{TeV}$

regions, these differences produce direct effects on the behavior of the $\mathrm{F}-\mathrm{B}$ multiplicity correlations.

Figure 3 shows $b_{\text {corr }}$ as a function of $\delta \eta$ : for central $(0<|\eta|<1)$ and fragmentation $(3<|\eta|<4)$ region, from here one can see the evolution of the strength correlation parameter, it shows a higher values for the soft processes respect to the hard ones. It is important to observe a faster saturation of the $b_{\text {corr }}$ than those calculated for soft processes. Comparisons short to long range correlations are shown in the bottom of the figure, where almost a scaling behavior of the $b_{\text {corr }}$ for soft processes is observed while for the hard processes it increases faster in the fragmentation region.

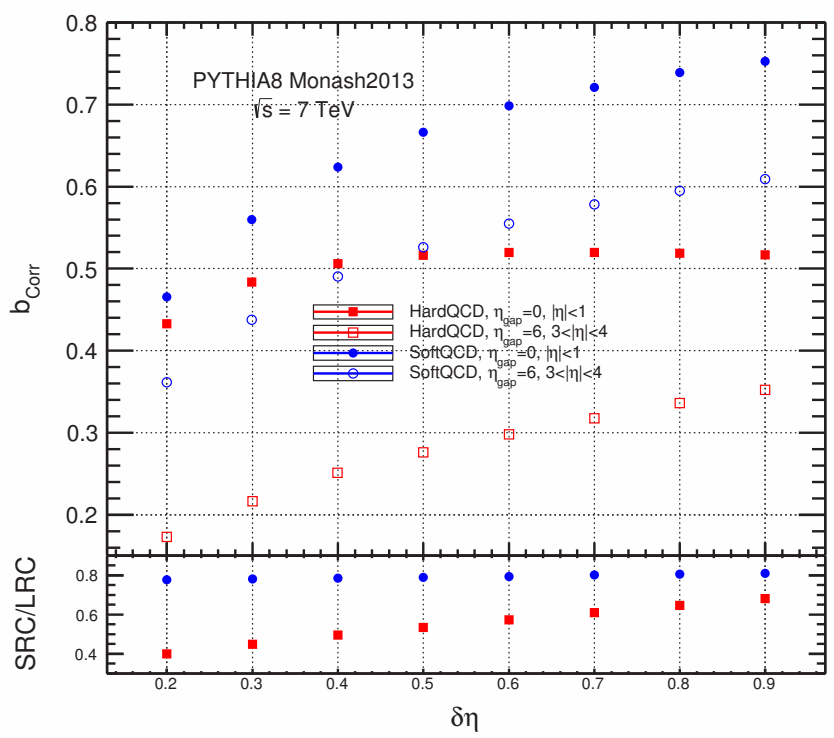

Figure 3: The correlation factor for $\mathrm{p}+\mathrm{p}$ collision at $\sqrt{s}=7 \mathrm{TeV}$, for soft and hard QCD processes in central and fragmentation pseudorapidity regions 


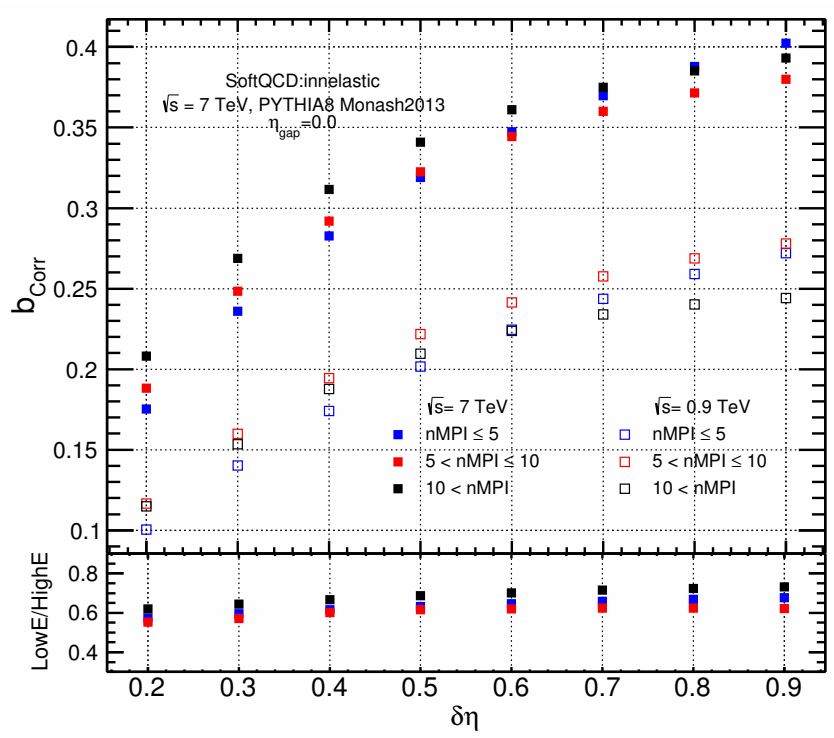

Figure 4: The correlation factor in terms of ranges of nMPI for $\mathrm{p}+\mathrm{p}$ collision at $\sqrt{s}=0.9,7 \mathrm{TeV}$.

\section{F - B Correlations and multiple parton interactions}

The multiple parton interactions is gaining interest since from the experimental point of view there are evidences of their existence and from theoretical side, there are calculations [9] which have been included in PYTHIA event generator. Figure 4 shows the correlations strength for two energies and for each one them, different ranges in the number of multiple parton interactions. The behavior in both cases is a continuous growth when the nMPI increase, however, comparing the correlation at low to high energy, one sees an almost flat behavior, it means, that $\mathrm{F}-\mathrm{B}$ multiplicity correlation shows a scaling with the energy. Combined effects on $b_{\text {corr }}$ coming from energy and nMPI could be understood from the relationship between these two parameters, since nMPI is modeled in PYTHIA with the damping parameter $\mathrm{p}_{0 \mathrm{~T}}[8]$ where this parameter is function of $p_{\mathrm{T}}$, and this last one depends directly on the collision energy.

\section{$4 \mathrm{~F}-\mathrm{B}$ correlations and color reconnection}

The CR has been proposed as an alternative mechanism to produce flow like effects in proton-proton collisions [10] where the direct variable that changes with the CR is the multiplicity distribution, it decreases when CR increases. CR has been taken to describe successfully the average transverse momentum of charge hadrons [11]. Figure 5 shows a reduction of the multiplicity when CR is included, and this will produce some effect on the strength in the F - B correlation multiplicity. 


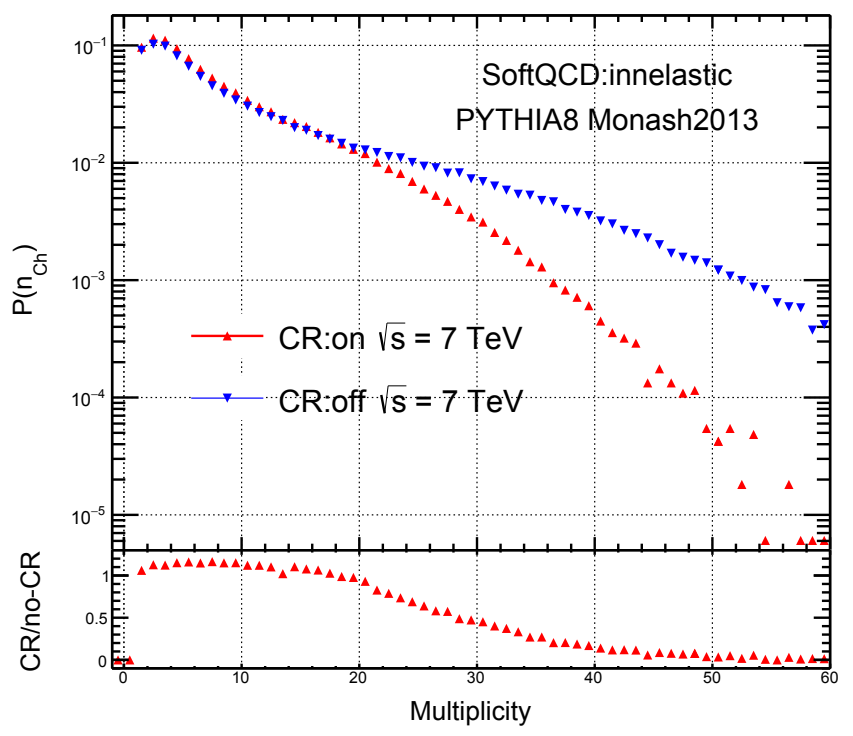

Figure 5: Multiplicity distribution of charge hadrons with and without color reconnection for $\mathrm{p}+\mathrm{p}$ collision at $\sqrt{s}=7 \mathrm{TeV}$.

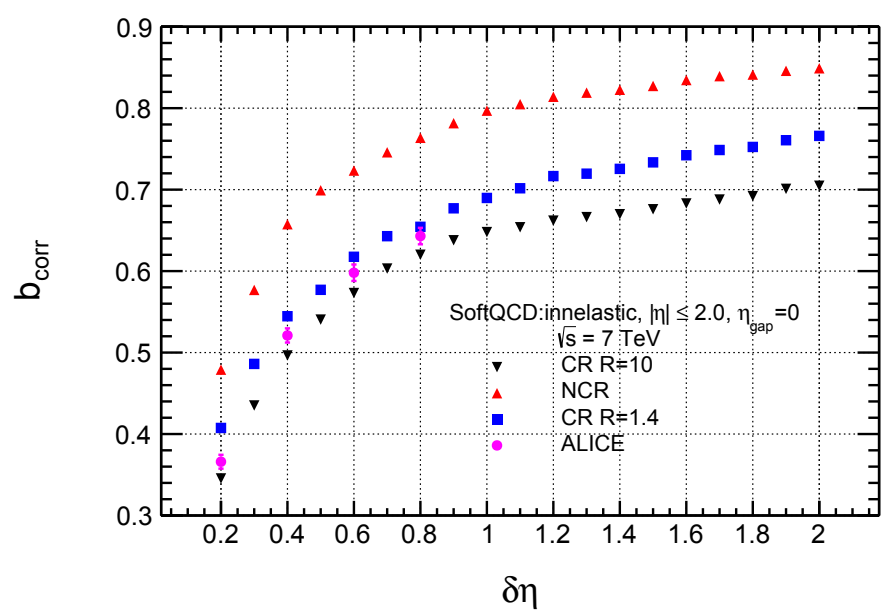

Figure 6: The correlation factor for Soft QCD processes in $\mathrm{p}+\mathrm{p}$ collisions at $\sqrt{s}=0.9,7 \mathrm{TeV}$. Comparison is done among different strength of CR and the experimental ALICE data.

Figure 6 shows the $b_{\text {corr }}$ as a function of the pseudorapidity width $\delta \eta$ at $7 \mathrm{TeV}$ for different values of the strength of the CR. It is observed that higher values of the CR reduce the multiplicity and also the $b_{\text {corr }}$. The possible range of values of CR strength is from 1.4 to 10 , and more precise value could be extracted from a fit of the experimental data which are shown in the same figure. However the CR 
is not the only possible effect present in the $b_{\text {corr }}$, then it is useful to make a more detailed analysis on the F-B correlation multiplicity.

\section{Conclusions}

In the present work we have compared the charged hadron production in the forward and backward, in central and fragmentation pseudorapidity regions. The analysis was done in $\mathrm{p}+\mathrm{p}$ collision at $\sqrt{s}=0.9$ and $7 \mathrm{TeV}$ using PYTHIA event generator, with the effects of the number of multiple parton interactions and different strength values of color reconnection, splitting the events into soft and hard QCD processes. The analysis of F - B multiplicity correlations shows a weak dependence with the number of multiple parton interactions while the energy produce almost scaling behavior of the correlations. However, when we took soft QCD processes a scaling between long and short range correlations is observed, meanwhile for hard processes a faster saturation of the $b_{\text {corr }}$ at central rapidity respect to the fragmentation region is observed. The color reconnection, which reduces the multiplicity also reduce the strength of the correlations, a combination of both numbers of multiple parton interactions and color reconnection could bring us an insight more detailed of hadron production mechanism in $\mathrm{p}+\mathrm{p}$ and other colliding systems.

\section{Acknowledge}

Partial support was received by DGAPA-PAPIIT and CONACYT projects IN109817 and 280362 respectively.

\section{References}

[1] British-French-Scandinavian Collaboration, M. Albrow et al.,Nucl. Phys. B145 305 (1978).

[2] UA5 Collaboration, K. Alpgard et al., Phys. Lett. B123 361 (1983).

[3] M. Derrick, K. Gan, P. Kooijman, J. Loos, B. Musgrave, et al.,Phys. Rev. D34 3304 (1986).

[4] E735 Collaboration, T. Alexopoulos, et al., Phys. Lett. B353 155 (1995).

[5] STAR Collaboration, B. Abelev et al., Phys. Rev. Lett. 103172301 (2009).

[6] ATLAS Collaboration, G. Aad et al.,JHEP 07019 (2012).

[7] ALICE Collaboration, Jaroslav Adam, et al, JHEP 1505097 (2015).

[8] T. Sjöstrand, S. Ask, J. R. Christiansen, R. Corke, N. Desai, P. Ilten, S. Mrenna, S. Prestel, C. O. Rasmussen, P. Z. Skands, Computer Physics Communications 191159 (2015.)

[9] Torbjörn Tjöstran and Maria van Zijl, Phys. Rev. D36, 2019 (1987).

[10] A. Ortiz Velasquez, P. Christiansen, E. Cuautle Flores, I. A. Maldonado Cervantes, and G. Paić, Phys. Rev. Lett. 111, 042001 (2013).

[11] ALICE Collaboration,B. Abelev Phys. Lett. B727 371-380 (2013). 